\title{
Under and over-adiabatic electrons through a perpendicular collisionless shock: theory versus simulations
}

\author{
P. Savoini ${ }^{1}$, B. Lembège ${ }^{1}$, V. Krasnosselskhik ${ }^{2}$, and Y. Kuramitsu ${ }^{2}$ \\ ${ }^{1}$ CETP/UVSQ, 32-40, Avenue de l'Europe, 78140 Vélizy, France \\ ${ }^{2}$ LPCE/CNRS, 3a, Avenue de la recherche scientifique, 45071 Orláns la Source, France
}

Received: 8 February 2005 - Revised: 11 October 2005 - Accepted: 17 October 2005 - Published: 23 December 2005

\begin{abstract}
Test particle simulations are performed in order to analyze in detail the dynamics of transmitted electrons through a supercritical, strictly perpendicular, collisionless shock. In addition to adiabatic particles, two distinct nonadiabatic populations are observed surprisingly: (i) first, an over-adiabatic population characterized by an increase in the gyrating velocity higher than that expected from the conservation of the magnetic moment $\mu$, and (ii) second, an underadiabatic population characterized by a decrease in this velocity. Results show that both nonadiabatic populations have their pitch angle more aligned along the magnetic field than the adiabatic one at the time these hit the shock front. The formation of "under" and "over-adiabatic" particles strongly depends on their local injection conditions through the large amplitude cross-shock potential present within the shock front. A simplified theoretical model validates these results and points out the important role of the electric field as seen by the electrons. A classification shows that both nonadiabatic electrons are issued from the core part of the upstream distribution function. In contrast, suprathermal and tail electrons only contribute to the adiabatic population; nevertheless, the core part of the upstream distribution contributes at a lower percentage to the adiabatic electrons. Underadiabatic electrons are characterized by small injection angles $\theta_{i n j} \leq 90^{\circ}$, whereas "over-adiabatic" particles have high injection angles $\theta_{i n j}>90^{\circ}$ (where $\theta_{i n j}$ is the angle between the local gyrating velocity vector and the shock normal).
\end{abstract}

Keywords. Space plasma physics (Charged particle motion and acceleration; Numerical simulation studies; Shock waves)

\section{Introduction}

The current understanding of the electron heating (for a review, see Scudder (1995) and the references herein) through

Correspondence to: P. Savoini

(philippe.savoini@cetp.ipsl.fr) a fast shock has emphasized three main points. First, the major increase in the electron temperature occurs within the ramp region (Bame et al., 1979). Second, the dominant process responsible for the shape of the electron distribution (and therefore the heating) mainly involves the action of the macroscopic fields, as demonstrated in detail by experimental measurements (Scudder et al., 1986a,b,c; Feldman et al., 1983) and confirmed in self-consistent numerical simulations by Savoini and Lembège (1994). Third, the main contribution for the perpendicular heating comes from the "reversible" inflation of the velocity space volume in the presence of magnetic forces (Feldman, 1985; Krauss-Varban, 1994; Scudder et al., 1986a; Scudder, 1995; Hull et al., 1998, 2001). This last point implies a transverse heating which preserves the value of $T_{e \perp} / B$ from upstream to downstream states.

Nevertheless, it has been observed that the adiabaticity may break down in supercritical shock waves. A comprehensive statistical study of electron heating versus various shock parameters has shown a moderate but systematic deviation from the adiabatic compression ratio (Schwarz et al., 1988). Scudder et al. (1986c) have also noted that the conservation of the fluid quantity $T_{\perp e} / B$ related to the magnetic moment $\mu$ requires conditions which are not obviously satisfied at collisionless shocks. Different mechanisms may be invoked. The most evident concerns the small-scale turbulence present at the shock front whose scattering could efficiently redistribute the energetic electrons, as shown numerically (Krauss-Varban, 1994; Krauss-Varban et al., 1995) and experimentally (Scudder et al., 1986c). Some experimental (Scudder et al., 1986c) and numerical results (Veltri et al., 1992) have even evidenced that wave particle interactions may "cool" the electrons rather than heat them.

Another possibility is the narrow ramp of certain collisionless shock (Newbury and Russell, 1996; Newbury et al., 1998; Walker et al., 1999), so that electrons do not follow the magnetic field variations (at least partially) and the magnetic moment is not conserved anymore. Even if the spatial scale of the magnetic and electric field variations inside the 
ramp is not too small, a certain percentage of the transmitted electrons can be demagnetized. As infered theoretically by Cole (1976) in the presence of an electric field gradient, the effective gyration frequency $\omega_{c e}^{e f f}$ differs from the magnetic gyro-frequency $\omega_{c e}$ by the value (given in the normalized coordinates used in this paper):

$\left(\widetilde{\omega}_{c e}^{e f f}\right)^{2}=\widetilde{\omega}_{c e}^{2}-\frac{d \widetilde{E}}{d \widetilde{x}}$,

where the electric field gradient along the $x$-direction $\frac{d \widetilde{E}}{d \widetilde{x}}$ is assumed to be constant within the transition region. This means that the scanning of the shock ramp by the electron gyromotion may drastically change according to the local strength of the $\frac{d \widetilde{E}}{d \widetilde{x}}$ gradient with respect to the local $B$ field, where "local" means that terms of Eq. 1 correspond to quantities "seen" locally by electrons.

This mechanism has been analyzed in details theoretically within the shock front (Gedalin et al., 1995a,c; Krasnosselskikh et al., 1995; Balikhin et al., 1998; Ball and Galloway, 1998). All these papers investigate the electron superadiabatic heating through the divergence of electron trajectories in the velocity space (i.e. using the Lyapounov coefficient $\gamma)$. These authors demonstrate that the cross-shock potential leads to an exponential expansion of close trajectories. In particular, a noticeable percentage of demagnetized electrons is always formed within the ramp itself and constitutes a good candidate for nonadiabatic heating through a shock front above a certain threshold. More recently, by using selfconsistent, full particle simulations, Lembège et al. (2003) have analyzed in detail the mechanisms responsible for the electron demagnetisation at the shock front, rather than focussing on their adiabatic/nonadiabatic behavior. They have confirmed the important role of the electrostatic field gradient along the shock normal in the demagnetisation processes but recovered only a partial (qualitative) agreement with the theoretical arguments proposed by Balikhin et al. (1998). Despite all these efforts, no detailed analysis has been performed until now on the criteria intrinsic to the transmitted electrons, in order to predict which part of the upstream electrons can become adiabatic or nonadiabatic.

According to the adiabatic theory (Northrop, 1963), the gyrating velocity of a particle moving under the influence of an increasing (decreasing) magnetic field alone will increase (decrease) in such way that the magnetic moment $\mu$ will be conserved or increased. Nevertheless, such a picture is incomplete, especially when electrostatic field gradients are self-consistently included in the shock front. In this case, the opposite behavior, leading to a decrease in the gyrating velocity, is also possible even for a strictly perpendicular shock, as shown in present results.

For oblique shocks, the macroscopic electrostatic field component parallel to the magnetic field accelerates incident solar wind electrons through the shock, resulting in a peak in $f(\boldsymbol{v})$ offset along $\boldsymbol{B}$ in the downstream direction relative to the plasma rest frame. Theoretically, it is simple to show that the parallel electric field strongly accelerates particles along the magnetic field and brings closer particles in the perpendicular velocity space. For example, suppose two particles have the velocity $v_{1}=v_{o}$ and $v_{2}=v_{o}+\delta v$, respectively. When including a potential $\Delta \phi$ in space along the $\boldsymbol{B}$ field, the conservation of the total energy allows us to obtain at the first order, the relation :

$\left(v_{2}-v_{1}\right)^{\text {final }}=\sqrt{\frac{m_{e}}{2 e \Delta \varphi}} v_{o}\left(v_{2}-v_{1}\right)^{\text {initial }}$.

Then, the electron trajectories in velocity space become closer depending on the value of the potential drop $\Delta \varphi$, leading to a reduction in the volume occupied by the electrons in the velocity space, i.e. corresponding to an electron cooling. Such a mechanism is well-known to operate along the magnetic field lines where particles are freely accelerated by the parallel electrostatic potential but in no way can be invoked in the strictly perpendicular case $(\boldsymbol{E} \perp \boldsymbol{B})$.

At this stage, in order to avoid any misunderstanding, it is important to define precisely the use of the words "adiabatic" and "nonadiabatic". Usually, nonadiabatic behavior means that the electron downstream temperature is higher than the electron heating expected from the magnetic field gradient only. If the time/space variations of the magnetic field are non negligeable within one gyro-period $\left(t_{\Delta B} \geq \tau_{c e}\right)$, the electric field speeds up the particle in such a way that the gyrating velocity exceeds the values obtained in the drift approximation, leading to nonadiabatic particles. Usually, there are two different ways to introduce the concept of adiabaticity which are not totally equivalent. First, as in Goodrich and Scudder (1984), adiabaticity is associated with the drift approximation or the conservation of the magnetic moment $\mu_{d s} / \mu_{u s} \approx 1$, where $\mu_{u s}$ and $\mu_{d s}$ are the upstream and the downstream magnetic momenta, respectively (individual particles approach). Second, adiabaticity is associated with heating, and conveys the increase in the internal energy that an assembly of particles should gain, owing to the conservation of $T_{\perp} / B$ (statistical approach).

The two different representations of the first adiabatic invariant must be used carefully. As a first step, hereinafter in this paper, we follow the first approach (individual trajectories) to define adiabatic $\left(\mu_{d s} / \mu_{u s} \sim 1\right)$ and nonadiabatic $\left(\mu_{d s} / \mu_{u s} \neq 1\right)$ electrons. Then, the primary goal of this paper is to investigate the respective role of the macroscopic fields gradient (magnetic and electric fields) and of the injection conditions into the shock front, in order to account for the final state of individual particles.

This paper is structured as follows. Section 2 contains a brief description of the 2-D full-particle simulations used to analyse the supercritical collisionless shock. In contrast with previous works based on an oblique shock (Lembège et al., 2003), the present analysis will consider a strictly perpendicular shock. Section 3 examines the time behavior of electron trajectories by using test particle simulations where fields profiles are issued from the 2-D full particle simulation results. Surprisingly, two types of nonadiabatic electrons, over-adiabatic and under-adiabatic, defined by $\mu_{d s} / \mu_{u s}>1$ 
and $<1$, respectively, are identified in the transmitted populations. Section 4 presents a simplified theoretical model in order to account the formation of the two types of nonadiabatic populations. A parametric study on the conditions of test particle simulations is presented in Sect. 5 in order to validate the theoretical model, while discussion and conclusions are summarized in Sect. 6.

\section{Numerical conditions}

In order to investigate the electron dynamic, a $2-1 / 2 D$, fully electromagnetic, relativistic particle code, using standard finite-size particle techniques, is used, whose details have been given in Lembège and Savoini (1992) and Savoini and Lembège (1994). The use of full-particle code is necessary, in order to obtain self-consistently the magnetic and electric field components present at the shock front, and in particular, the cross-shock potential which is expected to play a major role in the dynamics of transmitted nonadiabatic electrons.

Basic properties of the numerical code are summarized as follows. Nonperiodic conditions are applied along the $x$-direction within the simulation box and periodic conditions are used along the $y$-direction. The plasma simulation box lengths are $\widetilde{L}_{x}=6144$ and $\widetilde{L}_{y}=256$, which represents 102 and 4.3 inertial ion lengths $\left(\tilde{c} / \widetilde{\omega}_{p i}\right)$, respectively. The strictly perpendicular collisionless shock $\left(\Theta_{B n}=90^{\circ}\right)$, considered herein, will allow us to simplify both the theoretical model of Sect. 4 and the interpretation of the results (where $\Theta_{B n}$ is the angle between the upstream magnetic field $B_{O}$ and the shock normal).

Initial plasma conditions (i.e. upstream region) are summarized as follows (all physical parameters are normalised to dimensionless quantities “ $\sim$ "): light velocity $\widetilde{c}=3$, upstream magnetic field $\widetilde{B}_{o}=1.5$ (then, we have a ratio $\omega_{p e} / \omega_{c e} \approx 2$ ), temperature ratio between ion and electron population $T_{i} / T_{e}=1.58$, thermal velocity $\widetilde{v}_{t h e, x, y, z}=0.3$ for electrons and $\widetilde{v}_{t h i, x, y, z}=0.012$ for ions. The ratio $\beta_{e}$ of the electron kinetic to the magnetic pressure and the Alfvén velocity are $\beta_{e}=0.24$ and $\widetilde{v}_{A}=0.075$, respectively. The shock propagates in a supercritical regime $\left(M_{A}=5.14\right)$. A detailed study of the electrons dynamics and trajectories required one to use a high mass ratio. Nevertheless, at that time, a realistic mass ratio, $m_{i} / m_{e}=1840$, is still out of reach of 2-D, selfconsistent, full-particle simulations. Only 1-D shock simulations manage to include such a realistic mass ratio (Liewer et al., 1991; Scholer et al., 2003). As a compromise, a high mass ratio is used hereafter in this paper $\left(m_{i} / m_{e}=400\right)$. This value is high enough to separate the dynamics of electrons and ions, and to obtain more realistic space-charge effects and electric field gradients at the ramp than for a lower mass ratio. In terms of this lower mass ratio, we recover the main characteristics of a supercritical shock. Figure 1 shows cyclic self-reformation of the shock front, mainly due to the reflected ion population which accumulated over distance from the ramp until their density was high enough to form a new shock front (Lembège and Dawson, 1987;

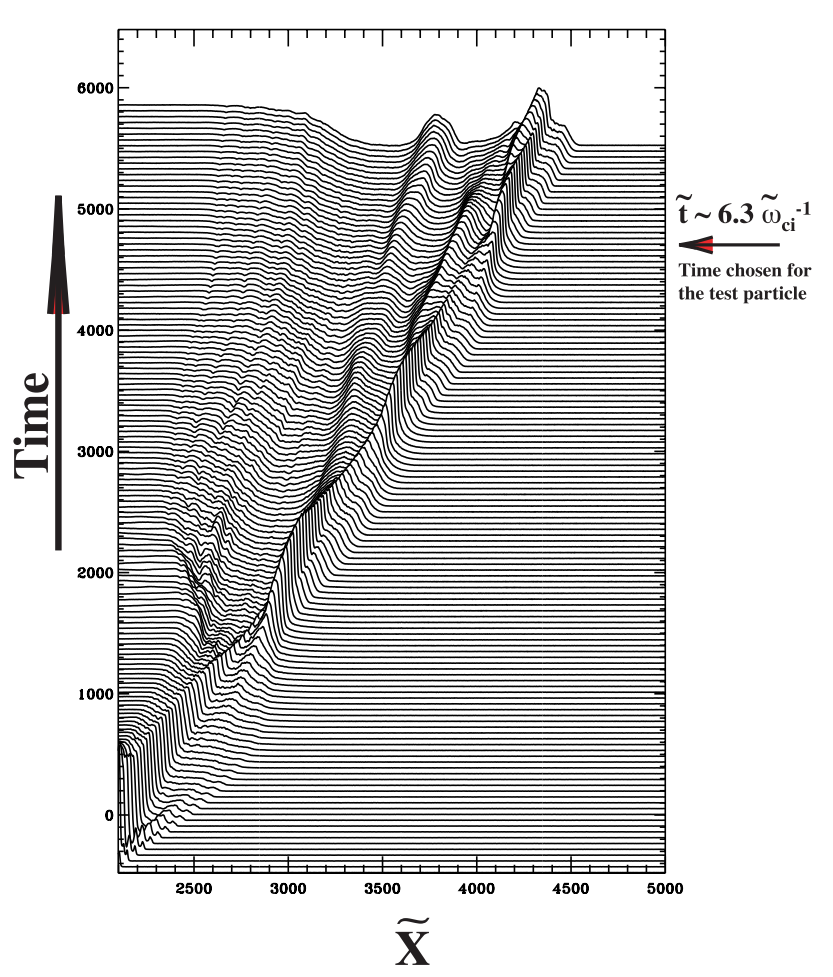

Fig. 1. Time evolution of the magnetic moment ratio $\mu / \mu_{u s}$ for the same selected particles of Fig. 3 (panels g, h, i) in absence of the electrostatic field component $\left(\widetilde{E}_{l x}=0\right)$. In order to emphasize the differences with Fig. 3, the same color code for the curves (black, red and blue) is used.

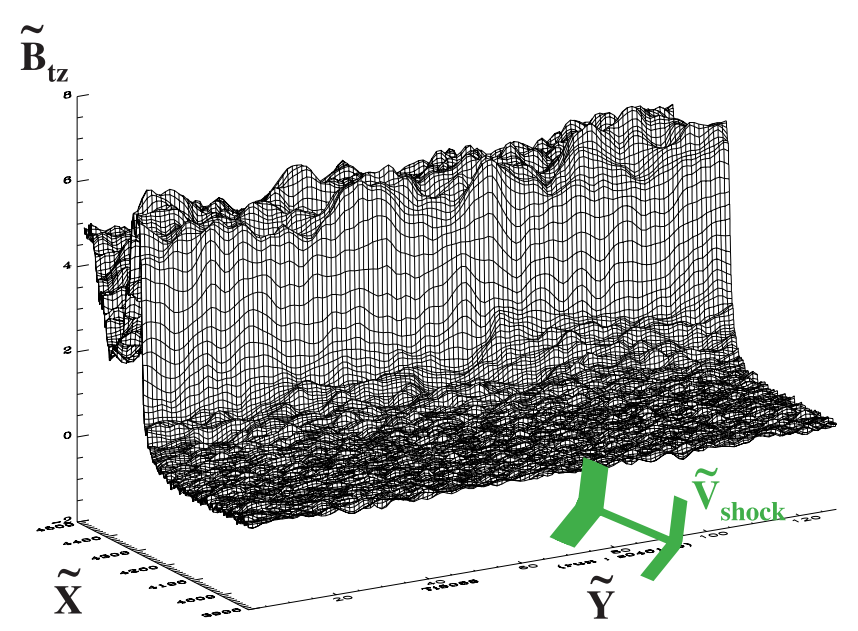

Fig. 2. Enlarged view of the main magnetic field component $\widehat{B}_{t z}(x, y)$ around the shock front at the time $\left(\widehat{t} \approx 6.3 \widehat{\omega}_{c i}^{-1}\right)$ chosen for the test particles simulations.

Lembège and Savoini, 1992; Scholer et al., 2003; Lee et al., 2004). Simultaneously, a shock front rippling, evidenced in Fig. 2, moving along the shock front, is the source of an additional nonstationarity. For the perpendicular case, the rippling has been identified as instabilities lying in the lower hybrid range and triggered by cross-field currents supporting 
the large field gradients at the front (Lembège and Savoini, 1992). These kind of instabilities are well-known to be of secondary importance on the global transmitted electron dynamic (for the formation of local, flat-topped distributions), both experimentally (Scudder et al., 1986a,b,c) and numerically (Lembège and Savoini, 1992) and will be excluded for simplicity in the present analysis.

A numerical test particle approach has been used based on all field components along the shock normal only and is summarized as follows. All secondary shock features which complicate the analysis have been eliminated by $y$-averaging all field components issued from 2-D, full-particle simulations (suppression of the shock front rippling) and by considering a given time only (suppression of the front selfreformation). In contrast with the self-consistent simulations where both particle and electromagnetic field dynamics are coupled, test particle simulations follow individual particles within pre-computed electromagnetic fields. Then, we only solve a particle pusher, following this set of first-order coupled differential equations:

$$
\begin{aligned}
& \frac{d \boldsymbol{r}}{d t}=\boldsymbol{v} \\
& \frac{d \boldsymbol{v}}{d t}=q[\boldsymbol{E}+\boldsymbol{v} \times \boldsymbol{B}],
\end{aligned}
$$

where $\boldsymbol{E}=\boldsymbol{E}(\tilde{x}, \tilde{y})$ and $\boldsymbol{B}=\boldsymbol{B}(\tilde{x}, \tilde{y})$. Presently, only $x$ profiles along the shock normal are concerned, as explained above. At late time $\left(\widetilde{t}=6.28 \widetilde{\omega}_{c i}^{-1}\right)$, a shock profile has been chosen from the 2-D, full particle simulation at the end of a self-reformation cycle, where the foot is almost absent, to avoid any interaction of the incoming electrons with the foot pattern. As a consequence, only the macroscopic fields at the ramp will control the time evolution of the particles through the shock front. Such a test particle method is quite appropriate to montain control of the initial particle locations both in the real and velocity space (phase space dependance analysis). We will see that these initial conditions have a strong impact on the electron dynamics.

\section{Numerical results: a single test particle approach}

At initial time, the (stationary) shock front is moving with a velocity $\widetilde{V}_{s h}=0.38$ along the $x$-axis (corresponding to $M_{A}=5.14$ in the 2-D full particle simulations). Electron test particles are at rest in the solar wind frame at some distance upstream from the shock front $(\tilde{X}=200 \widetilde{\triangle})$. It is important to point out that all test particles are at the same $x$ location. Electrons are distributed over a velocity sphere of radius $\widetilde{v}_{\text {shell }}=0.26$, so that only the phase angles differ from one particle to the other. As a reference, the value $\widetilde{v}_{\text {shell }}=0.52$ corresponds to the thermal velocity defined in the upstream electron distribution used in the full particle PIC simulation. As a consequence, all electrons see exactly the same shock profile, but their velocity components relative to the shock profiles and associated pitch angles will differ (phase angles effects).
Three distinct classes of transmitted electrons are identified (adiabatic, "over-adiabatic" and "under-adiabatic" particles namely, whose main features are summarized in Fig. 3. Panel (a), used as reference, shows the time evolution of the $x$ position of the particles (thin line) crossing the shock front (thick line). Panels (b) and (c) evidence the local magnetic and electrostatic fields seen by the particle versus time. Panels $(d, e, f)$ represent the corresponding time variation of the perpendicular velocity component. The change in $V_{\perp}$ can be evidenced by the amplitude of the oscillations, which shows that all electrons gain (or lose) perpendicular energy only during their crossing of the shock ramp $\left(5650 \widetilde{\omega}_{p e}^{-1} \leqslant \widetilde{t} \leqslant 5800 \widetilde{\omega}_{p e}^{-1}\right)$. During this time period, particles also undergo the effect of the electrostatic field present in the shock front. One striking feature is that no electron can be considered as demagnetized, as defined by Cole (1976), Balikhin et al. (1998) and Lembège et al. (2003), and so, even if the spatial widths of both the $\boldsymbol{E}$ and $\boldsymbol{B}$ fields at the shock ramp are comparable, $L_{E r} \approx L_{B r}$. Indeed, an enlarged view of the velocity space (not shown here) evidences that all particles regardless of whether they are "adiabatic", "under-" or "over-adiabatic" roughly suffer the same number of gyrations $(\approx 21)$ when crossing the ramp. The three selected electrons differ from each other by their magnetic moment variation between upstream and downstream regions, i.e. their ratio $\mu_{d s} / \mu_{u s}$, shown in panels ( $\mathrm{g}, \mathrm{h}$ and $\mathrm{i}$ ). The time range spent by the electron during the ramp crossing (defined in panel b) has been reported in all panels (colored orange area). The foot region is almost absent (the simulation time $\tilde{t}=6.28 \widetilde{\omega}_{p i}^{-1}$ has been chosen for such a reason) and only leads to an increase in the $v_{\perp}$ fluctuations of $\mu$, but without modifing the mean value of this parameter. The impact of this shock precursor will be not discussed in this paper.

The main differences between these particles can be described as follows:

(i) "Adiabatic electron (panel (g) of Fig. 3). As the particle goes into the shock front $\left(\widetilde{t} \approx 5650 \widetilde{\omega}_{p e}^{-1}\right)$, the fluctuations of the ratio $\mu / \mu_{u s}$ increases but the main value remains around 1. At the time the electron goes further into the downstream region $\left(\widetilde{t} \geqslant 5900 \widetilde{\omega}_{p e}^{-1}\right)$, it reaches a time-averaged value of $\approx 1.2$ until the end of the simulation.

(ii) "Over-adiabatic" electron (panel (h) of Fig. 3). This process does not seem efficient for the period $5650 \widetilde{\omega}_{p e}^{-1} \leqslant \widetilde{t} \leqslant 5690 \widetilde{\omega}_{p e}^{-1}$, which corresponds to the first part of the ramp. It is in this particular region (where $\nabla E>0$ ) that Lembège et al. $(2003,2004)$ have observed the demagnetization process of the incoming electrons for an oblique shock. We will see in Sect. 5 that such a process does not seem to be a good candidate to explain the present "over-adiabatic" electrons in a strictly perpendicular shock. Then, the magnetic moment ratio $\mu / \mu_{u s}$ continues to rise up almost to 4 in the second part of the ramp (where $\nabla E<0$ ) and reaches its maximum value $\left(\mu / \mu_{u s} \approx 9\right)$ when the particle leaves the overshoot and stabilizes around 7 , further downstream.

(iii) "Under-adiabatic" behavior (panel (i) of Fig. 3). We observe that the ratio $\mu / \mu_{u s}$ remains around 1, during the first part of the shock ramp. As the electron penetrates into 

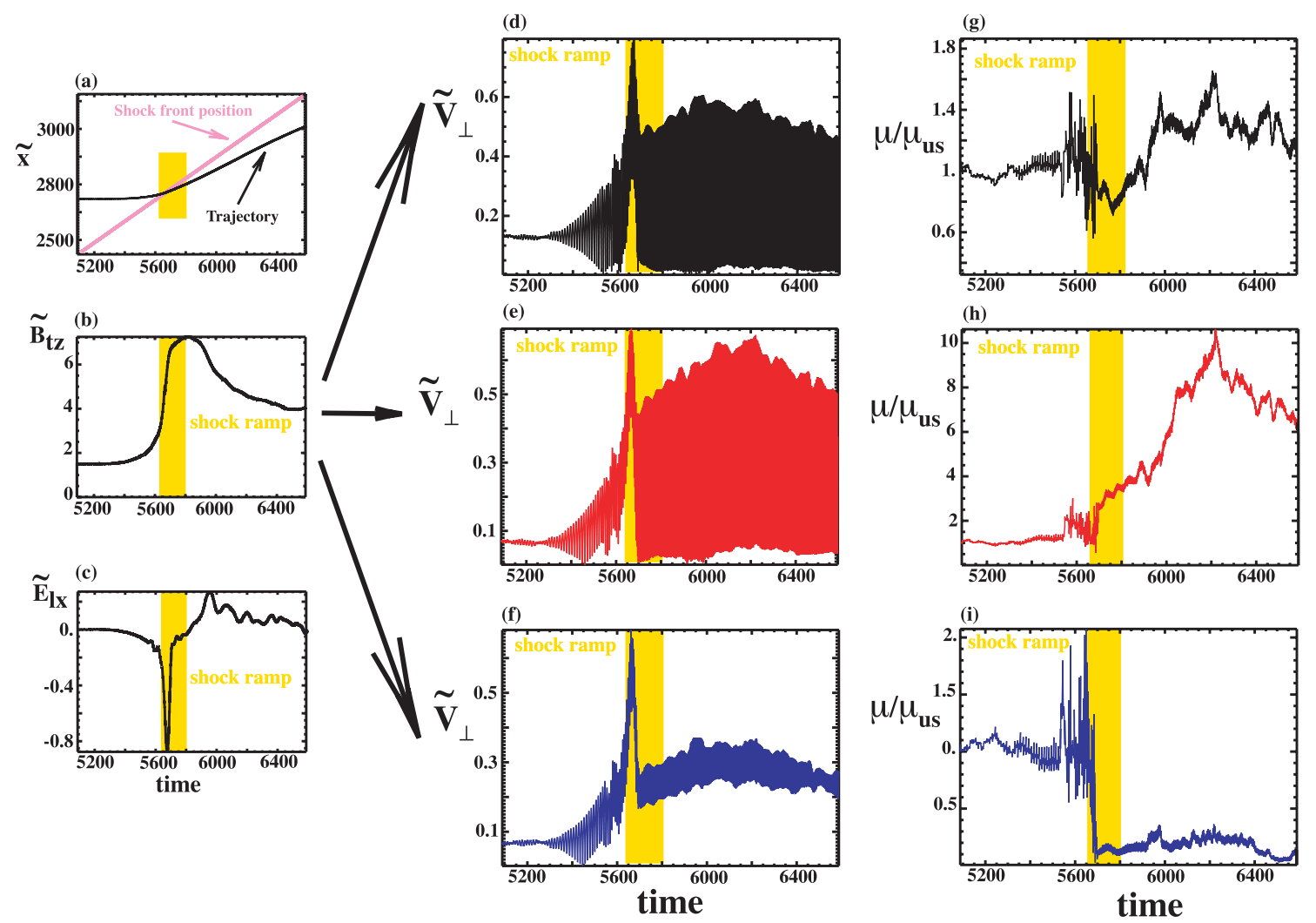

Fig. 3. Main characteristics versus time of selected electrons illustrating the three different types of transmitted electron populations (test particles simulation). All particles see the same macroscopic fields and have roughly the same trajectory. (a) $x$-coordinate of the particles (thin black line) and mean $x$-position of the shock front (thick pink line); (b) and (c) time history of the $\widetilde{B}_{t z}$ and $\widetilde{E}_{l x}$ fields seen by the different electrons. (d) to (f) and (g) to (i) show the time history of the perpendicular velocity and of the momentum ratio $\mu / \mu_{u s}$ respectively, where $\mu=m v_{\perp}^{2} / 2 B$ and $B$ is the local magnetic field seen by the particle. Black (panels d, g), red (panels e, h) and blue (panels f, i) colored plots are used in order to identify the so-called adiabatic, over-adiabatic and under-adiabatic electrons respectively defined by $\mu_{d s} / \mu_{u s} \approx 1,>1,<1$ ( $\mu_{u s}$ and $\mu_{d s}$ are, respectively, the upstream and the downstream value of the magnetic momentum).

the shock ramp at $\widetilde{t} \approx 5650 \widetilde{\omega}_{p e}^{-1}$, the magnetic momenta ratio suffers a drastical drop to $\mu / \mu_{u s} \approx 0.15$ which takes place during a short time range of several electron cyclotron periods $\left(\triangle \widetilde{t} \approx 10 \widetilde{\omega}_{c e}^{-1}\right.$, where $\widetilde{\omega}_{c e}^{-1}$ is the local electron gyrofrequency, i.e. within the first half of the ramp too). A close look of the perpendicular velocity (panel f) evidences that this drop is essentially driven by a decrease in the $V_{\perp}$ amplitude and not by a poor magnetic compression of a demagnetized electron. This indicates that the "under-adiabatic" process does not involve the magnetic field components, but rather the action of the space-charge electric field $E_{l x}$. Finally, as the particle goes further into the downstream region, the ratio $\mu / \mu_{u s}$ remains roughly constant. This particle is a good example of "under-adiabatic" behavior, although some similar particles exhibit the slight increase in their magnetic moment ratio $\mu / \mu_{u s}$ in the downstream region. For them, other downstream mechanisms have to be invoked which are out of the scope of this paper.

The comparison of "under-" and "over-adiabatic" electrons allows us to point out that the underlying processes are not spatially correlated. Under-adiabatic behavior takes place systematically within a very short time, within a fraction of the first part of the ramp, and then, can be related to the impact of the shock on the particle dynamic. On the other hand, the "over-adiabatic" behavior is a much slower process, occurring within both the ramp itself and a part of the downstream region. Obviously, the slow perpendicular energy changes involve other mechanisms. One possibility concerns the wave-particle interactions. It is out of the scope of this paper to investigate such wave activities. Nevertheless, it is important to bear in mind that in self-consistent 2-D, full particle simulations (even integrated along the $y$-direction), electric and magnetic fluctuations are present (along the $x$-direction). Such a fluctuation can efficiency scatter particles, as demonstrated by Karimabadi et al. (1992) and Krauss-Varban (1994) with the use of MonteCarlo simulations.

The "under-adiabatic" population is the most surprising feature of a strictly perpendicular collisionless shock. In order to investigate the role of the macroscopic electric field, another test particle run has been performed, in which the same electrons have been followed in absence of the longitudinal electric field $\left(\widetilde{E}_{l x}\right)$. Figure 4 shows, respectively, the time evolution of the magnetic momenta ratio $\mu / \mu_{u s}$ for the 


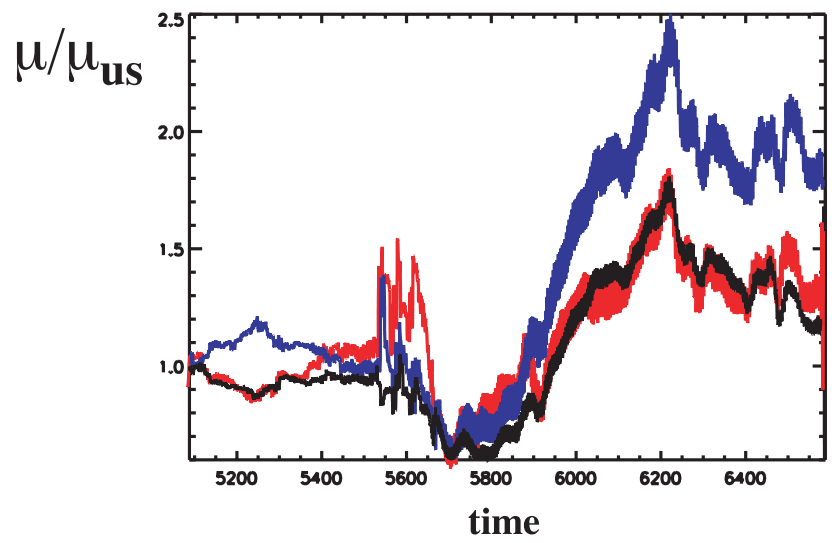

Fig. 4. Time evolution of the magnetic moment ratio $\mu / \mu_{u s}$ for the same selected particles of Fig. 3 (panels g, h, i) in absence of the electrostatic field component $\left(\widetilde{E}_{l x}=0\right)$. In order to emphasize the differences with Fig. 3, the same color code for the curves (black, red and blue) is used.

same "under-adiabatic" (blue line), "adiabatic" (black line) and "over-adiabatic" (red line) particles defined in Fig. 3. Of course, this simplified simulation neglects many features of a real collisionless shock. Nevertheless, some information can be deduced: First, the adiabatic particle exhibits no noticeable change in the ratio $\mu / \mu_{u s}$ in the two cases $\left(\widetilde{E}_{l x}\right.$ included/excluded). Even if the individual trajectory is not the same, the magnetic moment $\mu$ does not depend on the electric field component, in agreement with the adiabatic theory. Second, the "over-adiabatic" (red) particle appears to have an adiabatic behavior. This confirms that (i) the $\widetilde{E}_{l x}$ field can extract some electrons from the adiabatic "soup" to force these electrons to reach an overadiabatic level, and (ii) that any turbulence at the shock front is not necessary in order to obtain some overadiabatic electrons. Third, the most striking feature is that the under-adiabatic electron behavior totally disappears, which clearly evidences the key role of the electrostatic field component.

Since all electrons see the same shock profile, one has to determine how the electrostatic field contributes to the formation of these different electron populations, and to identify the main parameter connecting this field to the $\mu$ variation and to the velocity phase angle in the velocity space. For this purpose, a simple theoretical model is discussed in the next section.

\section{Theoretical model}

In a first approach, we follow Cole (1976), where the magnetic field is supposed to be constant. This restrictive approximation has the avantage of simplicity and allows some analytical solutions. This approach is valid as long as one considers only transmitted particles which suffer nonadiabatic processes on a time scale smaller than the magnetic field changes at the shock front. However, in contrast with Cole's model, the electric field gradient $\nabla E$ is not supposed (a)

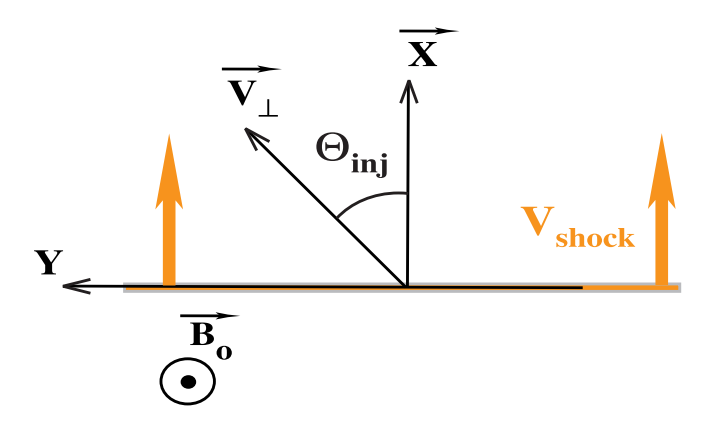

(b)
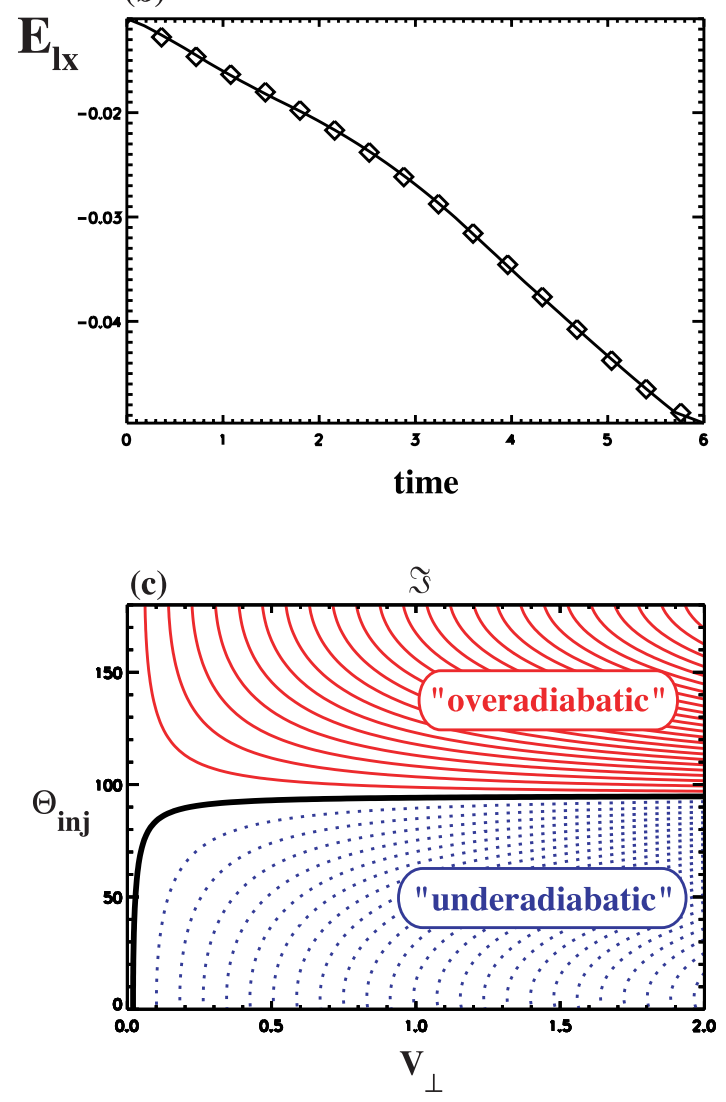

Fig. 5. (a) Sketch of the two-dimensional simulation shock front where the injection angle $\theta_{i n j}$ is defined between the normal $\boldsymbol{n}$ of the shock front (along $x$ ) and the local gyrating perpendicular velocity. The upstream magnetostatic field $\boldsymbol{B}_{o}$ is outside the simulation plane. (b) Enlarged view of time history of the electrostatic field component seen by the injected particles (thin line), within the ramp. A best fit is performed with a $4^{\text {th }}$ order polynom to get the $a_{n}$ coefficients used in our theoretical model and is represented by the squares. (c) plot of the quantity $\Im$ (Eq. 5) versus the injection angle $\theta_{i n j}$ and $v_{\perp}$ (gyrating velocity). The adiabatic line (thick line) separes the two nonadiabatic populations: (i) the "under-" (blue dashed lines) and the (ii) "over-adiabatic" electrons (red thin lines). 
to be constant at the ramp. Instead, the self-consistent time varying electric field seen by the particles (from PIC simulations) is injected into the analytical equations.

Consider the motion of a charged particle of mass $m$ and charge $q$ in an homogeneous magnetic field $\left(\boldsymbol{B}=B_{o} \boldsymbol{e}_{z}\right)$ and in an electric field gradient $\left(\boldsymbol{E}=E_{l} \boldsymbol{e}_{x}\right)$. The equation of motion is

$m \frac{d \boldsymbol{v}}{d t}=q\left[\sum_{n=0}^{n=N} a_{n} t^{n} \boldsymbol{e}_{x}+\boldsymbol{v} \times \boldsymbol{B}_{o}\right]$,

where the electric field is defined by the relation

$$
E_{l x}(t)=\sum_{n=0}^{n=N} a_{n} t^{n} .
$$

When $E_{l x}(t)$ is known and the magnetic field is assumed to be constant, this equation can be easily solved and allows us to determine all velocity components $\left(v_{x}, v_{y}\right.$ and $\left.v_{z}\right)$. As described in the Appendix, perpendicular velocities $v_{x}$ and $v_{y}$ are a function of two independent parameters: (i) their gyrating velocity $V_{\perp}$ (in the particle's frame) and (ii) $\theta_{i n j}$ defined in Fig. 5a which is the "injection" angle defined between the perpendicular velocity $\boldsymbol{v}_{\perp}$ and the normal $\boldsymbol{n}$ to the shock front at the time the particle hits the leading edge of the front. This angle is an important feature in the interaction of the electrons with fields at the shock front. Then, it is useful to compute the quantity $\Im$ (in the moving reference frame of the shock) which is the difference between the gyration velocity before and after the particles hit the shock front. Assuming that the magnetic field is constant, $\Im$ is simply the difference between the downstream and the upstream magnetic moment $\mu$ when transmitted particles undergo the effect of the electric field $E_{l x}$ only.

For the electron population, we have the relation

$\Im\left(v_{\perp}, \theta_{i n j}\right)=v_{\perp}^{2}-v_{o \perp}^{2}=\left(v_{e x}^{2}+v_{e y}^{2}\right)_{\text {gyrational }}-v_{o \perp}^{2}$.

Figure $5 b$ shows the electric field (thick line) seen by the particles as these cross the shock. This corresponds to a fraction of the first part of the ramp (Fig. 1), just after hitting the shock front, where electrons suffer a drastic change in their magnetic moment. The time evolution of the electric field of Fig. $5 \mathrm{~b}$ is fitted by a 4 th order polynom with chi-square goodness of fit equal to $1.5 \times 10^{-6}$. Using these values in Eq. (5), the dependence of $\Im$ versus $\theta_{i n j}$ and $v_{\perp}$ has been plotted in Fig. 5c. The values $\mathfrak{\Im}<0$ (dotted lines) and $\Im>0$ (thin lines) determine the region where "under-" and "over-adiabatic" electrons can be, respectively, identified. In this model, "adiabatic" particles are characterized by the thick line $(\Im=0)$ separating the two nonadiabatic populations. This figure allows one to stress three main points.

(i) First, the $\mathfrak{s}<0$ region spreads from $\theta_{i n j} \approx 0^{\circ}$ to $\theta_{i n j} \approx 90^{\circ}$. No "under-adiabatic" electrons exist for $\theta_{i n j}>90^{\circ}$ for any perpendicular velocity value. In contrast, the "overadiabatic" population is clearly separated from the "underadiabatic" one and is observed for higher injection angles $\left(\theta_{i n j}>90^{\circ}\right)$. (ii) The quantity $\Im\left(v_{\perp}, \theta_{i n j}\right)$ is almost independent of the $v_{\perp}$ parameter, except for the very small values. When $v_{\perp}<0.01$, all transmitted particles lie in the domain $\mathfrak{\Im}>0$, i.e. their perpendicular gyrating velocity increases. This can be understood if one keeps in mind that the quantity $\Im$ strongly depends on the particle gyromotion via the velocities $v_{x}$ and $v_{y}$, as described in the Appendix. When $v_{\perp}$ becomes very small, the gyromotion term in Eqs. (A2, A3) exhibits only the contribution of the electric field, and then are always positive.

(iii) Finally, it is worthwhile to note that adiabatic electrons are almost independent of the upstream perpendicular velocity for $v_{\perp} \geq 0.3$, and are defined only for injection angles around $90^{\circ}$. Nevertheless, the lack of magnetic field gradient in the model does not allow one to deduce relevant information on this particular population.

It is clear that such a simple model cannot describe in detail the electron dynamics at the shock front. Our model overestimates the "under-adiabatic" population as compared to the others, mainly because no magnetic compression is included $\left(\boldsymbol{B}_{o}=c t e\right)$. Nevertheless, in this paper, we appeal to the global behavior of the transmitted electrons rather than the exact amount of perpendicular gyrational kinetic energy gain (loss) at the shock front. At that point, the model can help us to emphasize the role of two parameters, namely the initial perpendicular velocity $v_{\perp}$ and the local injection angle $\theta_{i n j}$. This last quantity plays a key role in understanding the formation of "under-" and "over-adiabatic" electrons.

From this simple theoretical model, the two different populations strongly depend on the electron position of the Larmor gyro-radius circle at the time it hits the leading edge of the shock front (i.e. injection angle $\theta_{i n j}$ ), as reported in Fig. 5. To obtain a first insight, we have reported in Fig. 6 (bottom), the gyrating velocity (in the particle reference frame moving at the guiding center velocity) of the particles in Fig. 3 at the injection time. The $v_{\perp}$ velocity gyroradius decreases ("under-adiabatic" electron) or increases ("over-adiabatic" electron) during the first gyration within the ramp. As the particles go deeper into the ramp, they see a time increasing electric field. Consider an electron at rest in the upstream region whose trajectory is represented in Fig. 6. Then, in the dashed area (no dashed area) of the top panel, the electron moves in the direction (opposite direction) of the electrostatic field and then loses (gain) perpendicular kinetic energy. This process essentially occurs within the first gyration as the electron penetrates the shock ramp and is injected into another $v_{\perp}$ velocity gyroradius. Slightly later, the electron will see roughly the same field profiles and will gain/lose the same amount of energy through the magnetic and electric field gradients, and the resulting motion (increase or decrease of $v_{\perp}$ ) will be amplified.

\section{Multiple test particle approach}

Since the injection angle $\theta_{i n j}$ is an important parameter to account for the existence of an "under-adiabatic" particle, 


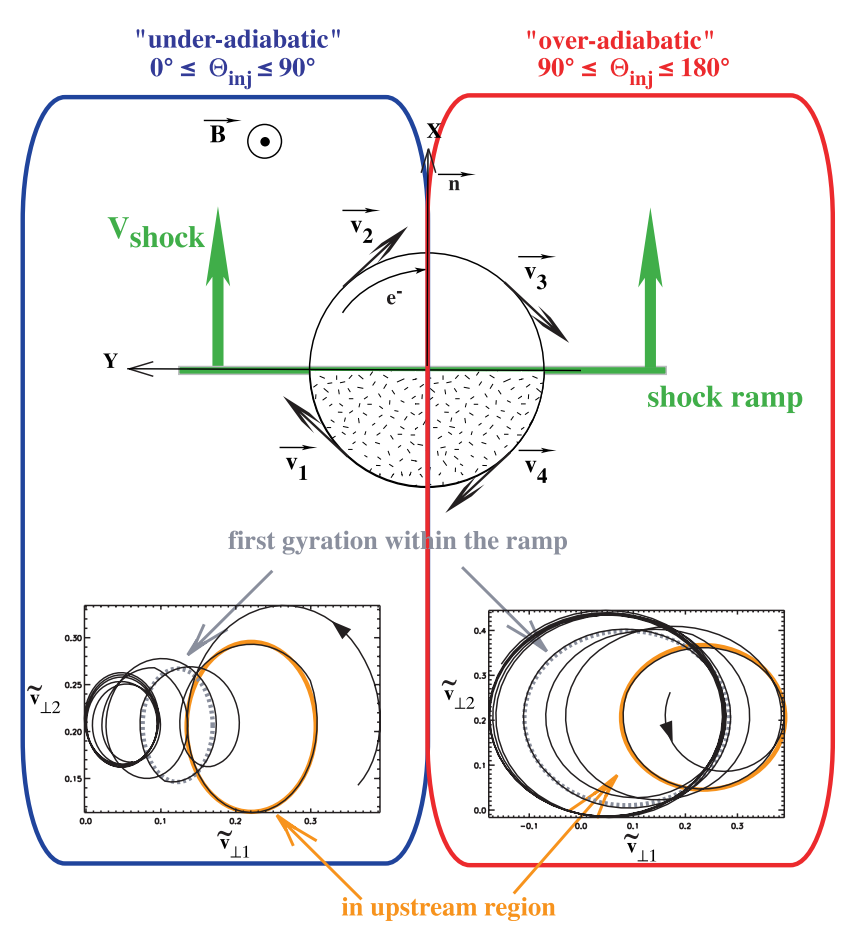

Fig. 6. Sketch of the electron trajectory (in the reference frame moving with the particle) when the particles hit the shock front (injection time) for the nonadiabatic behavior. All nonadiabatic particles with a perpendicular gyrating velocity (velocity $v_{1}$ and $v_{2}$ ) in the left part of the plot (blue frame) will suffer a "under-adiabatic" behavior from the electrostatic field present in the ramp. Conversely, the particles (velocity $v_{3}$ and $v_{4}$ ) in the right part of the plot (red frame) will be "over-adiabatic" under the action of the shock ramp electrostatic field. The green arrow represents the direction of the supercritical shock propagation. At the bottom, an enlarged view of the velocity space (several electron gyroperiods $\tau_{c e}$ ) is plotted before and after the particle hits the shock ramp. The gyromotion around the injection time (i.e. in the upstream region just before the electron enters the ramp) and the first full gyration performed within the ramp are indicated, respectively, by a thick orange line and a thick dotted blue line.

we have performed similar test particle simulations and analyzed a spherical shell (in the velocity space) of 580 individual electrons (Fig. 7a) instead of one test particle. A caveat is the fact that herein, the electrons do not fill the whole maxwellian upstream distribution, but instead only one particular part or shell (they have the same $\tilde{v}_{\text {shell }}$ ). The dependence on the shell radius $v_{\text {shell }}$ will be analyzed by launching a run with different shell radii as discussed later.

Our approach enables us to cover all different gyrating velocities from $\tilde{v}_{\perp o}=0$ to $\widetilde{v}_{\text {shell }}$, i.e. to analyze the impact of the phase angles in the velocity space. Indeed, in a strictly perpendicular shock, all particles which belong to the same ring in the velocity space will have the same $\tilde{v}_{\perp}$ when they hit the shock front. Simultaneously, since a ring covers different injection angles from $\theta_{i n j}=0^{\circ}$ to $180^{\circ}$, a comparison between the theoretical model (Fig. 5c) and the present simulation re-

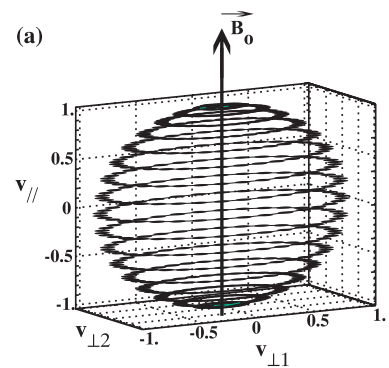

(b)
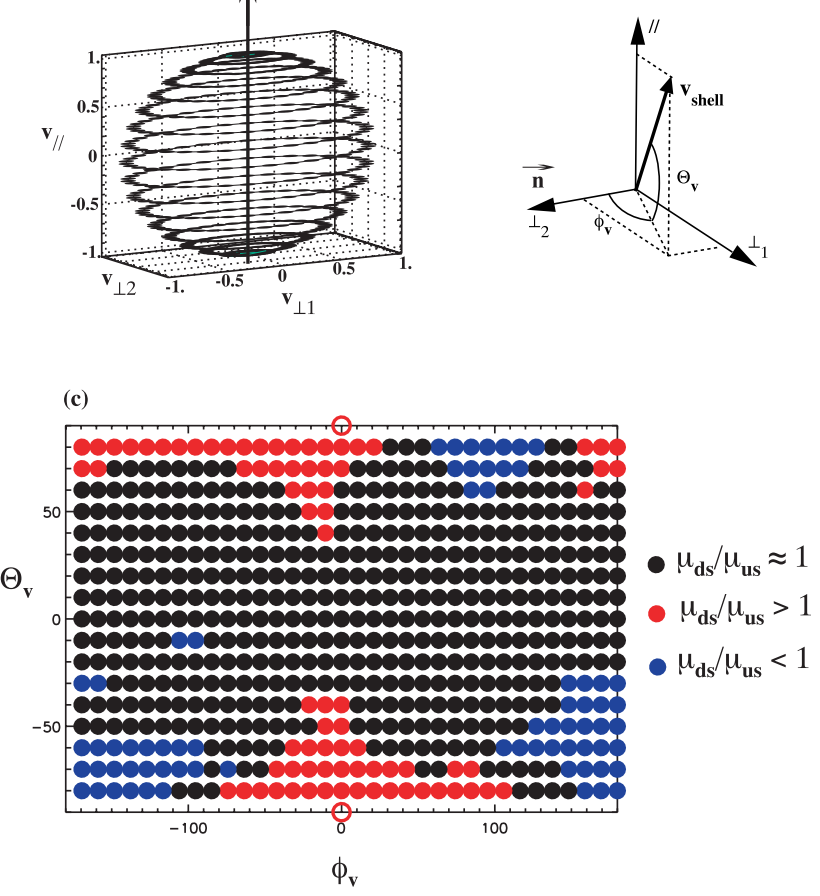

Fig. 7. (a) A spherical shell of 580 electrons (test particles) is located at $\widehat{x}=200 \triangle$, upstream from the front of the moving supercritical collisionless shock. The shell is aligned along the upstream magnetic field $\left(\boldsymbol{B}_{o}\right)$, (b) reference frame used for the spherical shell (radius $v_{\text {shell }}$ ), (c) initial upstream location $\left(\theta_{v}, \phi_{v}\right)$ of electrons versus their final state in the downstream region. This downstream state is indicated by the color code identifying the adiabatic (black), "under-" (blue) and "over-adiabatic" (red) electrons, as used in Fig. 1. We compute the mean value of $\mu$ in the downstream region over lots of gyrations, from the overshoot to the end of the simulation.

sults is directly possible. In order to simplify the representation of the particles in the velocity space, we have projected the spherical shell on the plane $\theta_{v}, \phi_{v}$, where the phase angles $\theta_{v}$ and $\phi_{v}$ are, respectively, defined by the angle between the vector $\boldsymbol{v}$ and the plane perpendicular to $\boldsymbol{B}$, and by the angle between the projection of $\boldsymbol{v}$ in the perpendicular plane and the direction of the shock normal $\boldsymbol{n}$ (see Fig. 7b).

A spherical shell of radius $\widetilde{v}_{\text {shell }}=0.26$ is used at the same position as the previous test particles of Sect. $3(\widetilde{X}=200 \widetilde{\triangle})$. For $M_{A} \approx 5.14$, the number of nonadiabatic electrons represents about $44 \%$ of the total transmitted electrons (for $\theta_{B n}=90^{\circ}$, no incoming electrons are reflected), with $26 \%$ "over-adiabatic" and 18\% "under-adiabatic".

Figure 7c represents locations of components of the upstream electrons (each dot stands for an individual particle). In this configuration, only 2 electrons have a velocity exactly aligned along the magnetic field $\boldsymbol{B}$ for exactly $\theta_{v}= \pm 90^{\circ}$ and $\phi_{v}=0^{\circ}\left(\widetilde{v}_{/ /}=\widetilde{v}_{\text {shell }}\right)$. Note that the colors used are similar to those in previous figures. Figure $7 \mathrm{c}$ allows one to emphasize three important points. 
(i) Even for a high Mach number $M_{A} \approx 5.14$, adiabatic electrons form almost half of the total transmitted electrons (about 56\%). These electrons are initially localized within the range $-50^{\circ} \leq \theta_{v} \leq 50^{\circ}$ for all values of the angle $\phi_{v}$. This means that an adiabatic electron is defined by a high perpendicular velocity component, or, in other words, by an initial pitch angle $\theta_{\widehat{B v}}=(\widehat{\boldsymbol{B}, \boldsymbol{v}})$ (defined by the angle between the magnetic field and the velocity vector) within the range $40^{\circ} \leq \theta_{B v} \leq 130^{\circ}\left(\theta_{\widehat{B v}}=90^{\circ}-\theta_{v}\right)$. It is important to emphasize that since the adiabatic population is not dependent on the angle $\phi_{v}$, it is also nondependent on the injection angle $\theta_{i n j}$ (linked to $\phi_{v}$ ). Then the initial pitch angle determines which particle will have an adiabatic or a nonadiabatic behavior but is not a relevant parameter to distinguish between the "under" and "over-adiabatic" populations.

(ii) The "over-adiabatic" electrons represent a smaller population. They are localized at high $\theta_{v} \quad\left(\left|\theta_{v}\right| \geq 50^{\circ}\right.$ and $-80^{\circ} \leq \phi_{v} \leq 80^{\circ}$ ), i.e. have a small, initial Larmor gyroradius (their velocity is mainly aligned along the magnetic field).

(iii) About $18 \%$ of all transmitted electrons exhibit an "under-adiabatic" behavior. The initial repartition of these electrons covers roughly the same broad range $\theta_{v}$ angles as the "over-adiabatic" population $\left(\theta_{v}>50^{\circ}\right)$. It is interesting to note that when a spherical shell is launched at $3 \widetilde{\triangle}$, before the present shell (not shown here), the repartition of the "under" and "over-adiabatic" populations in the velocity space $\left(\theta_{v}, \phi_{v}\right)$ is roughly reversed. This spatial range $(3 \widetilde{\triangle})$ corresponds to the distance covered by the shock front during half an electron gyro-period $\left(1 / 2 \tau_{c e}\right)$. During this time, the spherical shell rotates by $180^{\circ}$, which changes the locations of the nonadiabatic electrons within the velocity space when these hit the shock front (i.e. they are coming from the opposite side of the shell). This feature emphasizes the strong dependance of the nonadiabatic electrons versus the injection angle.

The understanding of the final downstream state requires one to investigate the local conditions, in terms of particle velocity and magnetic/electric field profiles seen by the particles at the injection time. These conditions are summarized in the Fig. 8 which shows characteristic parameters: (i) those defining the electron itself (i.e. the perpendicular velocity and associated quantities) and (ii) those describing the interaction between electrons and the electromagnetic fields (i.e. $\mu$ ). Panel (a) shows the locations that the different electron populations (color refers to their downstream adiabatic/nonadiabatic state) occupy within the perpendicular velocity plane $\left(\widetilde{v}_{\perp 1}, \widetilde{v}_{\perp 2}\right)$ at the injection time. Let us keep in mind that all particles have the same velocity $\left(\widetilde{v}_{\text {shell }}=0.26\right)$ and see exactly the same shock profiles (all magnetic and electric field components), whereas only the gyrating velocity around the magnetic field (the $\widetilde{v}_{\perp}$ component) varies from one particle to another (i.e. from one circle to another). The following considerations can be made:

- "Under-adiabatic" electrons (blue dots) are mainly localized in the $\widetilde{v}_{\perp 1}>0$ direction and are approximately distributed along the $\widetilde{v}_{\perp 2}$ direction. However, these do not exhibit any strong dependency versus $\widetilde{v}_{\perp 2}$ values (note that the shock velocity corresponds to about $\widetilde{v}_{\perp 2} \approx 0.38$ ). In summary, the "under-adiabatic" population has an initial perpendicular velocity spreading within a large $\widetilde{v}_{\perp}$ range $\left(0.05 \leqslant \widetilde{v}_{\perp} \leqslant 0.26\right)$. This means that $\widetilde{v}_{\perp}$ is definitively not relevant for identifying the "under-adiabatic" population.

- On the other hand, a large part of the "over-adiabatic" electrons (red dots) are localized around the origin of the perpendicular velocity space (small initial pitch angle) and form almost the first circle of our shell $\left(\widetilde{v}_{\perp} \approx 0.05\right)$.

- Finally, the adiabatic population (black dots) fills the whole velocity space and no clear separation can be made between adiabatic and nonadiabatic particles from this diagnostic.

The important role of the upstream pitch angle $\theta_{\widehat{B, v}}$ in separating adiabatic and nonadiabatic particle is illustrated from Fig. 7c. Nonadiabatic populations are essentially localized in the ranges $\theta_{v}>30^{\circ}$ and $\theta_{v}<-30^{\circ}$, whereas the adiabatic population lies more or less in the range $-30^{\circ} \leq \theta_{v} \leq 30^{\circ}$. Then the nonadiabatic (adiabatic) populations are characterized by a parallel component of the velocity $\boldsymbol{v}_{/ /}$higher than the perpendicular component $\boldsymbol{v}_{\perp}\left(\boldsymbol{v}_{\perp}>\boldsymbol{v}_{/ /}\right)$. However, the pitch angle $\theta_{\widehat{B, v}}$ is irrelevant to separate "under-" and "overadiabatic" populations.

In order to obtain a more quantitative insight into the nonadiabatic electrons, one must separate both populations ("under-" and "over-adiabatic" populations) at the injection time and not versus upstream parameters only. Figure $8 \mathrm{~b}$ plots the $\mu_{d s} / \mu_{u s}$ ratio versus the injection angle $\theta_{i n j}$. The most prominent effect concerns the "under-adiabatic" particles whose $\theta_{i n j}$ is clearly limited to the range $0^{\circ} \leq \theta_{i n j} \leq 90^{\circ}$, whereas the "over-adiabatic" population is essentially within the range $60^{\circ} \leqslant \theta_{i n j} \leqslant 180^{\circ}$. At this stage, we have to point out that "over-adiabatic" particles exhibit a continuous increase in their magnetic moment through the shock after the drop of $\mu / \mu_{u s}$ within the first half of the ramp (see Fig. 3h). Then the resulting downstream value $\mu_{d s}$ (and associated ratio $\mu_{d s} / \mu_{u s}$ ) cannot be used as a precise criterium since it does not maintain a history of the local injection conditions at the ramp (angle $\theta_{i n j}$ ). This may explain the presence of some "overadiabatic" electrons with an injection angle below $90^{\circ}$. More precisely, these electrons spread out over a wide angular range $\left(50^{\circ}<\theta_{i n j}<180^{\circ}\right)$, but their density decreases when approaching extrema angular values $\left(50^{\circ}\right.$ and $180^{\circ}$ ), as shown in Fig. 8 b.

On the other hand, adiabatic particles are observed for all possible angles from $\theta_{i n j}=0^{\circ}$ to $180^{\circ}$. Obviously, this population is totally independent of the angle $\theta_{i n j}$, which is coherent with the adiabatic scenario where the gyration energy is only proportional to the total magnetic field. 
(a)

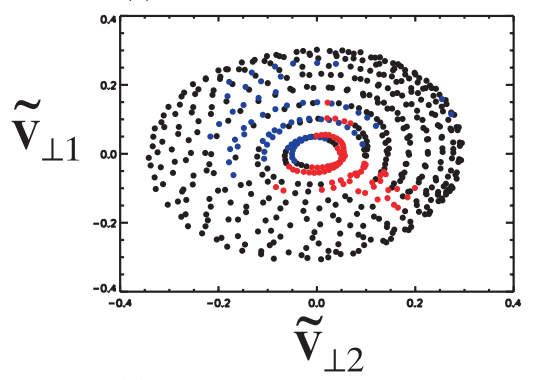

(b)

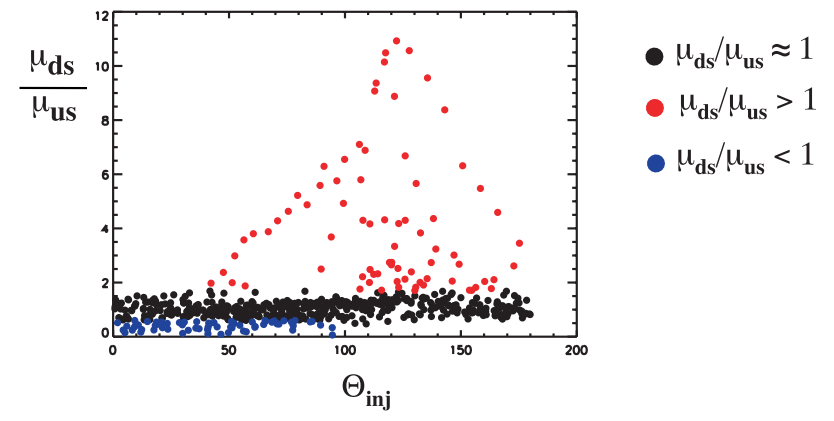

Fig. 8. (a) Projection of the shell in the local perpendicular velocity plane when the electrons hit the shock front (injection time). The same color code defined in Fig. 3 is used. Panel (b) shows magnetic moment ratio $\mu_{d s} / \mu_{u s}$ versus the injection angle $\theta_{i n j}$, defined by the angle between the gyrating velocity and the shock normal when the particle hits the ramp.

\section{Summary and discussion}

It is the first time, to our knowledge that, "under-adiabatic" particles are identified in strictly perpendicular shock conditions. Such a population cannot be explained theoretically by the energy conservation because we need an electric component parallel to the magnetic field to accelerate particles along $\boldsymbol{B}$ (Sect. 1) and another source mechanism needs to be invoked. As shown in this paper, the relevant parameter is the injection angle $\theta_{i n j}$, the angle between the gyrating velocity and the normal of the shock when the particle hits the ramp.

Lembège et al. (2003) have recently shown the importance of the electric field gradient amplitude within the shock ramp for the "demagnetization" of the incoming electrons. These authors observe that some transmitted electrons suffer a slowing down of their effective gyro-frequency within the first part of the ramp. Such demagnetized electrons have been invoked as potential candidates for the nonadiabatic population. However, these results are not recovered in this study, which shows that the electric field seen by the electrons varies over a much longer time scale (covering $\approx 21 \widetilde{\tau}_{c e}$ ) than the local electron gyration period. Then the existence of the present nonadiabatic electrons cannot be explained by the "demagnetization" process involved in the positive electric field gradient present in the first part of the ramp. This discrepancy may be explained as follows. The analysis of Lembège et al. (2003) has been made for an oblique shock and for a lower mass ratio $\left(m_{i} / m_{e}=42\right)$, and the magnetic momenta ratio diagnostic has not been used at that time. This is in contrast with the present analysis based on a strictly perpendicular shock and the use of a much higher mass ratio $\left(m_{i} / m_{e}=400\right)$. First, for an oblique shock, demagnetized electrons suffer a strong acceleration along the magnetic $\boldsymbol{B}$ field. Such an acceleration cannot occur in a strictly perpendicular shock, and the particle dynamic is totally different when $\theta_{B n}$ changes from $90^{\circ}$ to the oblique case $\left(\theta_{B n}<90^{\circ}\right)$. Second, the dynamics of the two populations (ions and electrons) are more clearly separated for a higher mass ratio. For clarifying the situation, we have performed an additional 2D PIC simulation of a strictly perpendicular shock, using now a lower mass ratio $\left(m_{i} / m_{e}=42\right)$ identical to that used by Lembège et al. (2003). Comparison between both simulations $\left(m_{i} / m_{e}=400\right.$ and 42$)$ shows that the maximum amplitude of the electric field gradient measured in the first part of the ramp (not shown here) is roughly the same $(\nabla E \approx 0.1)$. However, the time which during electrons see the electric field is higher for $m_{i} / m_{e}=400\left(\triangle_{t} \approx 15 \tilde{\tau}_{c e}\right.$, where $\tilde{\tau}_{c e}$ is the local gyro-period computed inside the first part of the ramp) than for the low mass ratio $\left(\triangle_{t} \approx 6 \widetilde{\tau}_{c e}\right.$ for $\left.m_{i} / m_{e}=42\right)$. As a consequence, the low mass ratio overestimates the demagnetization process in the final downstream state of the particles. Nevertheless, it is important to emphasize that all results, including the identification of adiabatic, over-adiabatic and under-adiabatic electrons, are fully recovered for the low mass ratio. Both kinds of simulations are qualitatively in good agreement and exhibit the same sensitivity to the injection angle $\theta_{i n j}$. However, a third question on "how injection criteria vary for an oblique shock", in order to evidence the two nonadiabatic populations, is still unanswered and is under active investigation at the present time.

\subsection{Impact of the velocity phase (for a given shell radius)}

In the test particle reference frame, all particles belonging to the same ring within the velocity space $\left(\widetilde{v}_{\perp 1}, \widetilde{v}_{\perp 2}\right)$ have the same $\widetilde{v}_{\perp o}$ at the time they hit the shock ramp. Moreover, since a ring covers different injection angles from $\theta_{i n j}=0^{\circ}$ to $180^{\circ}$, a comparison between the theoretical model (Fig. 5c) and the numerical results (Fig. 8b) is directly possible. The following conclusions can be made.

First, some systematic discrepancy appears concerning the adiabatic population even for a strictly perpendicular shock. In numerical results, the adiabatic population spreads out within the range $0^{\circ} \leq \theta_{i n j} \leq 180^{\circ}$, whereas theory predicts its existence mainly around $\theta_{i n j} \approx 90^{\circ}$ for all thermal velocity. As already pointed out in Sect. 5, our model is not appropriate to analyze this particular population.

Second, a relatively good agreement is obtained concerning the nonadiabatic electrons. From Fig. 8b, "underadiabatic" electrons are observed below $\theta_{i n j} \approx 100^{\circ}$ while "over-adiabatic" electrons are localized within a larger range $\left(50^{\circ} \leq \theta_{i n j} \leq 180^{\circ}\right)$. From Fig. 5c, "under-adiabatic" ("overadiabatic") electrons are defined for $\theta_{i n j} \leq 90^{\circ}\left(>90^{\circ}\right)$. At this point, it is important to emphasize the poor sensitivity of the 
critical value $\theta_{i n j}^{\text {crit }} \approx 90^{\circ}$ to the order of the polynom expansion of the electric field $\left(E_{l x}(t)=\sum_{n=0}^{n=N} a_{n} t^{n}\right)$. A plot of the quantity $\Im\left(v_{t h e}, \theta_{i n j}\right)$ (not shown here) for different values of $n(n>1)$ leads to the conclusion that the two opposite behaviors $(\mathfrak{I}<0$ and $\mathfrak{\Im}>0$, respectively) are always present for any value of $n$. Only the value of the cutoff $(\Im=0)$, separating the two populations, has to be refined to fit the numerical results. As evidenced, both "under-" and "over-adiabatic" populations may be considered as a common feature of particles injected into an electric field gradient.

Third, the function $\Im\left(v_{\perp}, \theta_{i n j}\right)$ (Fig. $\left.5 \mathrm{c}\right)$ is nearly insensitive to the perpendicular velocity for an injection angle in the range $0^{\circ} \leq \theta_{i n j} \leq 60^{\circ}$. Since many particles of the spherical shell are lying in this injection angle range, the number of "under-adiabatic" electrons is high, as evidenced by the number of blue dots present for $\theta_{i n j} \leq 60^{\circ}$ in the Fig. 8b. Conversely, for $60^{\circ} \leq \theta_{i n j} \leq 90^{\circ}$, less particles are able to check simultaneously $\theta_{i n j}$ and $\widetilde{v}_{\perp}$ conditions, and an important reduction of blue dots is observed in Fig. 8b.

We therefore conclude that the most important parameter which identifies the "under-adiabatic" electrons is the local injection angle at the time they hit the shock ramp. Even if the physical picture inferred from our oversimplified model cannot directly apply to a real collisionless shock wave, the deduced arguments provide useful insight into the physics and clarify the role of the injection angle $\theta_{i n j}$ to separate the two nonadiabatic populations.

\subsection{Impact of the thermal velocity (variation of the sphere radius)}

A given spherical shell (fixed radius) allows us to investigate all possible injection angles, but is intrinsically limited in identifying which part of the upstream distribution function is responsible for the different transmitted electron populations. Then, in order to cover the whole upstream electron distribution function, we have simulated a series of shells with different radii from 0.01 to 1.3 . Figure 9 summarizes the results and plots the percentage of adiabatic and nonadiabatic populations versus the radius of the shell $\widetilde{v}_{\text {shell }}$. The vertical dashed line represents the value of the thermal velocity of the upstream electron distribution function $\left(\widetilde{v}_{\text {the, us }} \approx 0.52\right.$ ). Clearly, two different velocity domains can be defined: (i) $\widetilde{v}_{\text {shell }}<\widetilde{v}_{\text {the, us }}$, where most transmitted electrons exhibit a nonadiabatic behavior and (ii) $\widetilde{v}_{\text {shell }}>\widetilde{v}_{\text {the, us }}$ where almost all particles have approximately an adiabatic behavior (we reach an asymptotic slope to $\approx 100 \%$ ). This indicates that the influence of the electrostatic field potential on transmitted electrons decreases as the shell velocity increases. In other words, the breakdown of adiabaticity is mainly controlled by all electrons (independently of the phase or injection angle) located in the body of the distribution, in agreement with previous theoretical works (Cole, 1976; Gedalin et al., 1995b; Balikhin et al., 1998). Then suprathermal electrons $\left(\widetilde{v}_{\text {shell }}>\widetilde{v}_{\text {the, us }}\right)$ and electrons in the distribution tail do not contribute to the adiabaticity breakdown.

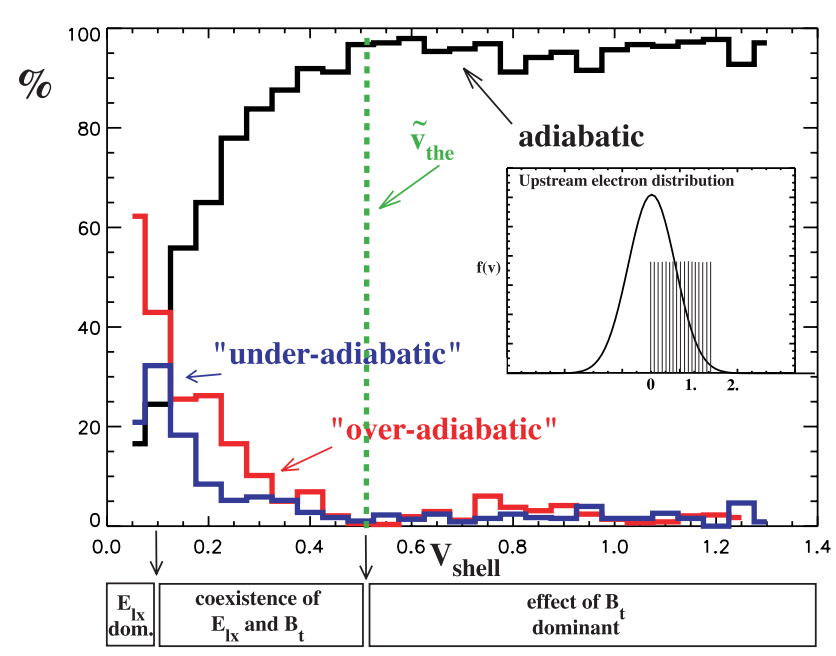

Fig. 9. Percentage of adiabatic, "under-" and "over-adiabatic" electrons versus the shell radius $v_{\text {shell }}$. The same color code (black, red, blue) defined in Fig. 3 is applied for the three electron populations. The middle panel illustrates the upstream electron distribution function and the different values of $v_{\text {shell }}$ used for scanning the distribution. The vertical green dashed line stands for the thermal velocity of the upstream electron distribution function $\left(\widetilde{v}_{t h e}\right)$. Three different domains can be evidenced: (i) at low velocity, the electrostatic field dominates and no under-adiabatic electrons are observed, (ii) at intermediate values, both electric and magnetic field influence the electron dynamic and the three populations are identified, and finally, (iii) for the larger velocity, the impact of the electric field becomes insignificant, where all transmittted electrons suffer an adiabatic compression.

Finally, a closer inspection of Fig. 8 shows that the percentage of "under-adiabatic" ("over-adiabatic") electrons decreases (increases) drastically as $\widetilde{v}_{\text {shell }}$ approaches to 0 . This feature can be explained with the simple theoretical model of Sect. 3, which evidences that electrons cannot lose gyration energy for very small upstream gyrating velocities. In other words, the impact of the electric field becomes dominant for very small $\widetilde{v}_{\perp}$ (through the terms $C_{o}$ and $C_{1}$, see Eq. (A2) and (A3) in the Appendix), and the resulting energization is always equal or higher than the adiabatic level. According to this result, the deep core of the electron distribution function (i.e. $\widetilde{v}_{\perp} \leqslant 0.01$ ) is not able to produce "under-adiabatic" particles, as evidenced by the decrease of this population in Fig. 9.

We have clarified the origin of nonadiabatic behavior of transmitted electrons by the cross-shock potential at a perpendicular shock. This effect is a direct result of the injection conditions of the electrons in the strong electric field within the shock ramp. It is important to keep in mind that the processes responsible for the adiabaticity breakdown have been presently analyzed for a strictly perpendicular shock, and for stationary field profiles, taking into acccount the spatial variations of the field components along the shock normal direction only. Other intrinsic features, such as the largescale nonstationarity of the shock front (self-reformation 


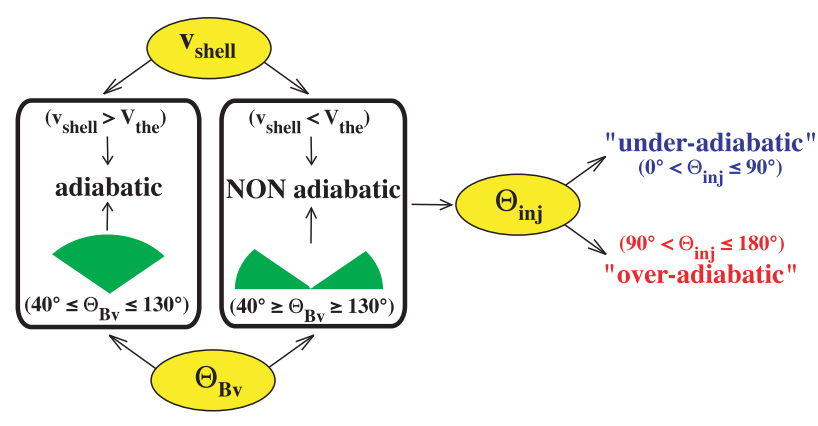

Fig. 10. Synoptic of the three key parameters (yellow disks) involve in the adiabatic/nonadiabatic behavior classification. The pitch angle $\theta_{B v}$ defined approximately two different behaviors, as illustrated by the green portion of the $(\vec{B}, \vec{v})$ plane: (i) for $40^{\circ} \leq \theta_{B v} \leq 130^{\circ}$, individual $\mu$ are conserved while (ii) for $40^{\circ} \geq \theta_{B v}, \theta_{B v} \geq 130^{\circ}$, they are nonconserved. Among transmitted nonadiabatic electrons, injection angle $\theta_{i n j}$ clearly separates the "under-" and the "over-adiabatic" behavior which appears under and over $90^{\circ}$, respectively.

along $x$ ) and the small-scale nonstationarity/nonuniformity of the shock front (rippling along $y$ ) observed in full particle simulations (see Figs. 1 and 2), have been removed on purpose in our test particle simulations. This means that turbulence within the shock front (induced by local microinstabilities, as observed by Scholer et al. (2003)) is not a necessary ingredient for the breakdown of adiabaticity. Thus, the higher-order irreversible dissipation provided by wave particle interactions has been removed; in particular, wave particle effects, which cool the electron distribution function (by filling the void of inaccessibility) (Scudder et al., 1986b,a,c) has been excluded.

Analysing in detail not only the time evolution of the field components seen by the electrons but also the quantities characterizing these particles, has led us to the following conclusions. First, a positive magnetic field gradient can only lead to the formation of adiabatic and/or "over-adiabatic" electrons. Only the electric field gradient in the ramp can be responsible for the formation of "under-adiabatic" electrons. Second, three different classes of nonadiabatic/adiabatic particles can be defined for directly transmitted electrons, depending of three key parameters as sketched in Fig. 10. The first key parameter which allows one to distinguish these classes is the relative electron velocity amplitude with respect to the thermal velocity of the upstream distribution. Only the core of the upstream electron distribution function $\left(v \leq v_{\text {the }}\right)$ can have a nonadiabatic behavior, and even in this case, adiabatic particles are always observed with a velocity not aligned to the $B$ field (i.e. a pitch angle in the range $40^{\circ} \leq \theta_{\widehat{B v}} \leq 130^{\circ}$ ). Suprathermal and tail electrons $\left(v>v_{\text {the }}\right)$ have an adiabatic behavior. The second key parameter for separating adiabatic/nonadiabatic electrons is the upstream pitch angle of the particle for a given shell radius. Electrons with a parallel velocity component higher (lower) than the perpendicular component will be nonadiabatic (adi- abatic). In summary, an appropriate combination of these parameters, relative particle velocity versus the thermal velocity of the upstream distribution function and the upstream pitch angle, may provide an indication as to which part of the upstream distribution function will contribute to the adiabatic/nonadiabatic electron populations. However, no upstream parameter will allow one to separate under- and overadiabatic electrons. Therefore, a third key parameter is necessary: the injection angle $\theta_{i n j}$ of the electron velocity precisely defined at the time the electron hits the shock ramp (injection time). We observe that particles with a small injection angle, $\theta_{i n j} \leq 90^{\circ}$, have mainly a "under-adiabatic" behavior, whereas those with high injection angles, $\theta_{i n j}>90^{\circ}$, have an "over-adiabatic" behavior.

We have focussed the present analysis on the individual electron dynamics (trajectory and velocity features) and not on their statistical variation in the velocity space (distribution function). Therefore, the analysis of adiabaticity breakdown through the notion of temperature (statistical approach) is under active investigation and will be presented in a forthcoming paper.

\section{Appendix A Equation of motion in the theoritical model}

In our model, we consider a constant magnetic field along the $z$ axis $\left(\boldsymbol{B}=B_{o} \boldsymbol{e}_{z}\right)$, and an electric field aligned along the $x$ axis $\left(\boldsymbol{E}=E_{l} \boldsymbol{e}_{x}\right)$. The electric field gradient is also along this direction, which is the direction of the shock front normal. In order to compare further with our numerical simulations, we use a time power series of Nth order to fit the electric field (obtained in our 2-D full particle simulation) seen by the electrons through the ramp.

The equation of motion of a charged particle of mass $m$ and charge $q$ in this electromagnetic field configuration is:

$$
m \frac{d \boldsymbol{v}}{d t}=q\left[\sum_{n=0}^{n=N} a_{n} t^{n} \boldsymbol{e}_{x}+\boldsymbol{v} \times \boldsymbol{B}_{0}\right],
$$

where the electric field is given by:

$$
E_{l x}(t)=\sum_{n=0}^{n=N} a_{n} t^{n}
$$

Since the magnetic field is assumed to be constant, this equation can be easily solved and allows us to determine the velocity components $\left(v_{x}, v_{y}\right.$ and $\left.v_{z}\right)$ versus the initial parameters. In a perpendicular configuration, no acceleration is possible along the $z$ direction and we have $v_{z}=c t e=v_{o z}$. Along the other directions, the equation of motion yields to the system

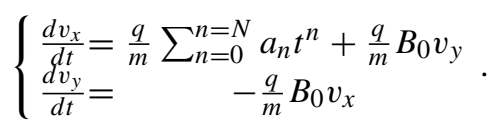


Since we are interested herein only in electrons $(q=-e$ and $\left.m=m_{e}\right), v_{y}$ becomes the solution of the second order differential equation:

$$
\frac{d^{2} v_{y}}{d t^{2}}+\omega_{c}^{2} v_{y}=+\frac{e}{m_{e}} \omega_{c} \sum_{n=0}^{n=N} a_{n} t^{n},
$$

where $\omega_{c e}=e B_{o} / m_{e}$ is the electron cyclotron frequency. Then, the perpendicular velocity components are defined by:

$$
\begin{array}{cc}
v_{e x}= & -\left[v_{o \perp} \cos \theta_{o}-C_{o}\right] \sin \left(\omega_{c} t\right) \\
& -\frac{1}{\omega_{c}}\left[C_{1}+\rho_{c} \omega_{c}^{2} \sin \theta_{o}\right] \cos \left(\omega_{c} t\right) \\
& -\frac{e}{m} \sum_{n=0}^{n=6}\left(B_{o} C_{n}+a_{n}\right) \frac{t^{n+1}}{n+1} \\
+ & v_{o \perp} \sin \theta_{o}+\frac{1}{\omega_{c}}\left[C_{1}+\rho_{c} \omega_{c}^{2} \sin \theta_{o}\right]
\end{array}
$$

and

$$
\begin{aligned}
& v_{e y}=\left[v_{o \perp} \cos \theta_{o}-C_{o}\right] \cos \left(\omega_{c} t\right) \\
& -\frac{1}{\omega_{c}}\left[C_{1}+\rho_{c} \omega_{c}^{2} \sin \theta_{o}\right] \sin \left(\omega_{c} t\right) \\
& +\sum_{n=0}^{n=N} C_{n} t^{n},
\end{aligned}
$$

with the coefficients $C_{n}$ solution of Eq. (A1). These coefficients can be easily computed, assuming that electrons have a cyclotronic gyroradius $\rho_{c e}=v_{\perp} / \omega_{c e}$ in the upstream region, so that:

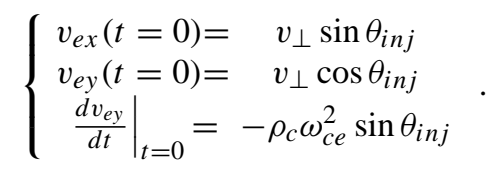

In this equation set, $\theta_{i n j}$ is the so-called injection angle, the angle between the perpendicular velocity and the $x$-axis at the time the particle hits the shock front (injection time). After some algebra, we can express the $C_{n}$ parameters versus the electric field coefficients $a_{n}$ seen by the injected electrons as a series

$$
\begin{cases}C_{n}=-\frac{1}{\omega_{c}} a_{n}-\frac{1}{\omega_{c}^{2}}(n+2)(n+1) C_{n+2} & \\ C_{n}=-\frac{1}{\omega_{c}} a_{n} & \mathrm{n} \leq \mathrm{N}-2 \\ & \mathrm{n}>\mathrm{N}-2,\end{cases}
$$

where $N$ is the power of the time series defining the electric field and $n$ an integer varying from 1 to $N$.

Equations (A2) and (A3) point out the following results. Three parts contribute to the perpendicular velocity components: (i) a modified oscillating periodic motion, (ii) an acceleration or deceleration, depending on the coefficients $C_{n}$ along the $x$-axis and (iii) an accelerated/decelerated drift along the $y$-axis. It is out of the scope of this paper to investigate the particle acceleration within a temporal electric field gradient, so we focus only on the oscillating part. We also note that when all $a_{n}$ coefficients are zero, then the $C_{n}$ coefficients vanish, and Eqs. (A2) and (A3) reduce to the wellknown uniform gyro-motion with the frequency $\omega_{c e}$ (i.e. no electric field $\nabla E_{l}=0$ ).

These equations evidence some perturbations of the particle gyrating velocity (i.e. $v_{\perp}$ ) as the particle enters the electric field gradient region. Indeed, in the particle reference frame moving with the drift velocity, particles perform gyrations with a velocity varying according to the values of the coefficients $C_{0}$ and $C_{1}$. Consequently, the first adiabatic invariant $\mu$ is modified. The coefficients $C_{0}$ and $C_{1}$ are obtained by looking for the best fit of the electric field seen by injected electrons, as shown in Sect. 4 . As far as the theoretical model is concerned (first part of the ramp) only, the increase in the electric field also leads to two important features: (i) an acceleration along the $x$-axis due to the difference between the foreward (opposite direction of $\boldsymbol{E}$ ) and downward (direction of $\boldsymbol{E}$ ) particle gyromotion, and (ii) an acceleration in the $\boldsymbol{E} \times \boldsymbol{B}$ direction due to the Lorentz force, as described by Eqs. (A2) and (A3).

Acknowledgements. This work was initiated under the auspices of the International Space Science Institute (ISSI, Bern, Switzerland) which is thanked for its hospitality and financial support. Simulation runs have been made at the IDRIS center (Orsay).

Topical Editor T. Pulkkinen thanks a referee for his/her help in evaluating this paper.

\section{References}

Balikhin, M. A., Krasnosselskikh, V., Woolliscroft, L. J. C., and Gedalin, M. A.: A study of the dispersion of the electron distribution in the presence of $\mathrm{E}$ and $\mathrm{B}$ gradients: Application to electron heating at quasi-perpendicular shocks, J. Geophys. Res., 103, 2029-2040, 1998.

Ball, L. and Galloway, D.: Electron heating by the cross-shock electric potential, J. Geophys. Res., 103, 17 455-17 465, 1998.

Bame, S. J., Asbridge, J. R., Gosling, J. T., Halbig, M., Paschmann, G., Sckopke, N., and Rosenbauer, H.: High temporal resolution observations of electron heating at the bow shock, Space Sci. Rev., 23, 75-92, 1979.

Cole, K. D.: Effects of crossed magnetic and spatially dependent electric fields on charged particle motion, Planet Space Sc., 24, 515-518, 1976.

Feldman, W. C.: Electron velocity distributions near collisionless shocks, Collisionless shocks in Heliosphere: Reviews of current research, edited by: Tsurutani, T. B. and Stone, R. G., Geophysical monograph series 35, American Geophysical Union, 195205, 1985.

Feldman, W. C., Anderson, R. C., Bame, S. J., Gary, S. P., Gosling, J. T., McComas, D. J., and Thomsen, M. F.: Electron velocity distributions near the Earth's bow shock, J. Geophys. Res., 88, 96-110, 1983.

Gedalin, M., Balikhin, M., and Krasnosselskikh, V.: Electron heating in quasi-perpendicular shocks, Adv. Space Res., 15, 225233, 1995a.

Gedalin, M., K. G., Balikhin, M., Krasnosselskikh, V., and Woolliscroft, L. J. C.: Demagnetization of electrons in inhomogeneous E, B: Implications for electron heating in shocks, J. Geophys. Res., 100, 19911-19918, 1995b.

Gedalin, M., M., K. G., Balikhin, M., Krasnosselskikh, V., and Woolliscroft, L. J. C.: Demagnetization of electrons in electromagnetic field structure, typical for quasi-perpendicular collisionless shock front, J. Geophys. Res., 100, 9481-9488, 1995c.

Goodrich, C. C. and Scudder, J. D.: The adiabatic energy change of plasma eletrons and the frame dependence of the cross-shock 
potential at collisonless magnetosonic shock waves, J. Geophys. Res., 89, 6654-6662, 1984.

Hull, A. J., Scudder, J. D., Frank, L. A., and Paterson, W. R.: Electron heating and phase space signatures at strong and weak quasiperpendicular shocks, J. Geophys. Res., 103, 2041-2054, 1998.

Hull, A. J., Scudder, J. D., Larson, D. E., and Lin, R.: Electron heating and phase space signatures at supercritical, fast mode shocks, J. Geophys. Res., 106, 15 711-15 733, 2001.

Karimabadi, H., Krauss-Varban, D., and Terasawa, T.: Physics of pitch angle scattering and velocity diffusion 1 . Theory, J. Geophys. Res., 97, 13 853-13 864, 1992.

Krasnosselskikh, V. V., Balikhin, M. A., Gedalin, M. E., and Lembège, B.: Electron dynamics in the front of the quasiperpendicular shocks, Adv. Space Res., 15, 239-245, 1995.

Krauss-Varban, D.: Electron acceleration at nearly perpendicular collisionless shocks 3. Downstream distributions, J. Geophys. Res., 99, 2537-2551, 1994.

Krauss-Varban, D., Pantellini, F. G. E., and Burgess, D.: Electron dynamics and whistler waves at quasi-perpendicular shocks, Geophys. Res. Lett., 22, 2091-2094, 1995.

Lee, R. E., Chapman, S. C., and Dendy, R. O.: Numerical simulations of local shock reformation and ion acceleration in supernova remnants, Astrophys. Journal, 604, 187-195, 2004.

Lembège, B. and Dawson, J. M.: Self-consistent study of a perpendicular collisonless and nonresistive shock, Phys. Fluids, 30, 1767-1788, 1987.

Lembège, B. and Savoini, P.: Non-stationarity of a 2-D quasi-perpendicular supercritical collisonless shock by selfreformation, Phys. Fluids, AA, 3533, 1992.

Lembège, B., Savoini, P., Balikhin, M., Walker, S., and Krasnoselskikh, V.: Demagnetization of transmitted electrons through a quasi-perpendicular collisionless shock, J. Geophys. Res., 108, SMP 20-1 SMP 20-18, 2003.

Lembège, B., Giacalone, J., Scholer, M., Hada, T., Hoshino, M., Krassnoselskikh, V., Kucharek, H., Savoini, P., and Terasawa, T.: Selected problems in collisionless-shock physics, Space Sci. Rev., 110, 161-226, 2004.

Liewer, P. C., Decyk, V. D., Dawson, J. M., and Lembège, B.: Numerical studies of electron dynamics in oblique quasiperpendicular collisionless shock waves, J. Geophys. Res., 96, 9455-9465, 1991.
Newbury, J. A. and Russell, C. T.: Observations of a very thin collisionless shock, Geophys. Res. Lett., 23, 781-784, 1996.

Newbury, J. A., Russel, C. T., and Gedalin, M.: The ramp widths of high mach number, quasi-perpendicular collisionless shocks, J. Geophys. Res., 103, 29 581-29 593, 1998.

Northrop, T. G.: The adiabatic motion of charged particles, WileyInterscience, New-York, 1963.

Savoini, P. and Lembège, B.: Electron dynamics in two and one dimensional oblique supercritical collisionless magnetosonic shocks, J. Geophys. Res., 99, 6609-6635, 1994.

Scholer, M., Shinohara, I., and Matsukiyo, S.: Quasi-perpendicular shocks: Length scale of the cross-shock potential, shock reformation, and implication for shock surfing, J. Geophys. Res., 108 (A1), SSH4-1, 2003.

Schwarz, S. J., Thomsen, M. F., Bame, S. J., and Stansberry, J.: Electron Heating and the Potential Jump Across Fast Mode Shocks, J. Geophys. Res., 93, 12 923-12 931, 1988.

Scudder, J. D.: A review of the physics of electron heating at collisionless shocks, Adv. Space Res., 15, 181-223, 1995.

Scudder, J. D., Mangeney, A., Lacombe, C., Harvey, C. C., and Aggson, T. L.: The resolved layer of a collisionless, high $\beta$, supercritical, quasi-perpendicular shock wave, 2. Dissipative fluid electrodynamics, J. Geophys. Res., 91, 11 053-11 073, 1986 a.

Scudder, J. D., Mangeney, A., Lacombe, C., Harvey, C. C., Aggson, T. L., Anderson, R. R., Gosling, J. T., Pashmann, G., and Russell, C. T.: The resolved layer of a collisonless, high $\beta$, supercritical, quasi-perpendicular shock wave. 1. Rankine-Hugoniot geometry, currents, and stationarity, J. Geophys. Res., 91, 11 019-11 052, $1986 b$.

Scudder, J. D., Mangeney, A., Lacombe, C., harvey, C. C., Wu, C. S., and Anderson, R. R.: The resolved layer of a collisionless, high $\beta$, supercritical, quasi-perpendicular shock wave, 3. Vlasov electrodynamics, J. Geophys. Res., 91, 11 075-11 097, $1986 c$.

Veltri, P., Mangeney, A., and Scudder, J. D.: Reversible electron heating vs. Wave-particle Interactions in Quasi-perpendicular shocks, Nuovo Cimento, 15, 607-619, 1992.

Walker, S. N., Balikhin, M. A., Alleyne, H. S. C. K., Baumjohann, W., and Dunlop, M.: Observations of a very thin shock, Adv. Space Res., 24, 47-50, 1999. 